\title{
Urine Eosinophils for Acute Interstitial Nephritis
}

\author{
Melbeth Lusica, MD¹, Helbert Rondon-Berrios, $\mathrm{MD}^{1 *}$, Leonard Feldman, $\mathrm{MD}^{2}$
}

${ }^{1}$ Renal-Electrolyte Division, Department of Medicine, University of Pittsburgh School of Medicine, Pittsburgh, Pennsylvania; ${ }^{2}$ Division of General Internal Medicine, Department of Medicine, John Hopkins University School of Medicine, Baltimore, Maryland.

The "Things We Do for No Reason" series reviews practices which have become common parts of hospital care but which may provide little value to our patients. Practices reviewed in the TWDFNR series do not represent "black and white" conclusions or clinical practice standards, but are meant as a starting place for research and active discussions among hospitalists and patients. We invite you to be part of that discussion.

Acute interstitial nephritis (AIN) is an important cause of acute kidney injury (AKI) in the hospital setting. However, the diagnosis of AIN is challenging because of its nonspecific clinical manifestations and the invasiveness of kidney biopsy, the gold standard for diagnosis. Urine eosinophils (UEs) emerged several decades ago as a noninvasive alternative for diagnosing AIN. Initial studies found UEs had a significant diagnostic value, but these studies had small sample sizes, and the diagnosis of AIN was made on clinical grounds only, without biopsy confirmation. In this article, we review the literature on the diagnostic value of UEs in the diagnosis of AIN.

\section{CASE REPORT}

A 62-year-old woman with type 2 diabetes mellitus, systemic hypertension, coronary artery disease, and obesity is admitted for AKI found on routine laboratory testing. She has been taking amoxicillin and doxycycline for left leg cellulitis the past 5 days, but improvement has been minimal. On admission, blood pressure is $120 / 74 \mathrm{~mm} \mathrm{Hg}$, and heart rate is 89 beats per minute. Serum creatinine level is increased, from $0.7 \mathrm{mg} / \mathrm{dL}$ at baseline to $3.6 \mathrm{mg} / \mathrm{dL}$ on admission. Complete urinalysis reveals $1+$ protein and presence of white blood cells and isormorphic red blood cells. No casts or crystals are seen. Given the possibility of AIN, UE testing is ordered. UEs are positive at $25 \%$. Does this result significantly increase the patient's posttest probability of having AIN?

\section{WHY YOU MIGHT THINK ORDERING URINE EOSIN- OPHILS IN THE EVALUATION OF AIN IS HELPFUL}

AKI occurs in more than 1 in 5 hospitalizations and is associated with a more than 4 -fold increased likelihood of

\footnotetext{
*Address for correspondence and reprint requests: Helbert Rondon-Berrios, MD, Renal-Electrolyte Division, Department of Medicine, University of Pittsburgh School of Medicine, A915 Scaife Hall, 3550 Terrace St, Pittsburgh, PA 15213; Telephone: 412-647-3120; Fax: 412-647-6222; E-mail: rondonberriosh@upmc.edu

Received: August 29, 2016; Revised: October 27, 2016; Accepted: November 1, 2016
}

2017 Society of Hospital Medicine DOI 10.12788/jhm.2737 in-hospital mortality at 21 days. ${ }^{1} \mathrm{AIN}$ is an important cause of AKI and has been found in $6 \%$ to $30 \%$ of AKI patients who had biopsies performed. ${ }^{2-4} \mathrm{AIN}$ is characterized by infiltration of inflammatory cells in the kidney interstitium and is more commonly caused by drugs, especially betalactam antibiotics, and less commonly by autoimmune or systemic diseases and infections. As the signs and symptoms of AIN are nonspecific, and the gold-standard test is renal biopsy, diagnosticians have sought a noninvasive test, such as UEs.

In 1978, Galpin et al. ${ }^{5}$ found that UEs comprised $10 \%$ to $60 \%$ of urine white blood cells in 9 of 9 patients with methicillin-induced interstitial nephritis; 6 of the 9 had biopsy-proven AIN. In 1980, Linton et al. ${ }^{6}$ found UEs in 6 of 9 patients with drug-induced AIN; 8 of the 9 had biopsy-proven AIN. In 1986, Nolan et al. ${ }^{7}$ reported that, compared with Wright stain, Hansel stain was more sensitive in visualizing UEs; they did not use biopsy for confirmation. Wright-stain detection of UEs is limited by the variable staining characteristics of "eosinophilic" granules in body fluids other than blood. With Hansel stain, UEs are readily identified by their brilliant red-pink granules. These 3 small studies helped make UEs the go-to noninvasive test for assessing for AIN. ${ }^{8}$

\section{WHY THERE IS LITTLE REASON TO ORDER URINE EOSINOPHILS IN PATIENTS WITH SUSPICION FOR AIN}

While initial studies indicated UEs might be diagnostically helpful, subsequent studies did not. In 1985, Corwin et al. ${ }^{9}$ used Wright stain and found UEs in 65 of 470 adults with AKI. Only 9 (14\%) of the 65 had a diagnosis of AIN, which was made mostly on clinical grounds. These findings showed that UEs were produced by other renal or urinary tract abnormalities, such as urinary tract infections, acute tubular necrosis, and glomerulonephritis. In a second study, Corwin et al. ${ }^{10}$ found that Hansel stain (vs Wright stain) improved the sensitivity of UEs for AIN diagnosis, from $25 \%$ to $62.5 \%$. Sensitivity was improved at the expense of specificity, as Hansel stain was positive in other diagnoses as well. The AIN diagnosis was not confirmed by kidney biopsy in the large majority of patients in this study. Lack of confirmation by biopsy, the gold-standard diagnostic test, was a methodologic flaw of this study and others.

Sutton $^{11}$ reviewed data from 10 studies and found AIN could not be reliably excluded in the absence of UEs (only 19 of 32 biopsy-confirmed AIN cases had UEs present). In addition, Ruffing et al. ${ }^{12}$ used Hansel stain and concluded 
TABLE. Urine Eosinophils in the Diagnosis of Acute Interstitial Nephritis

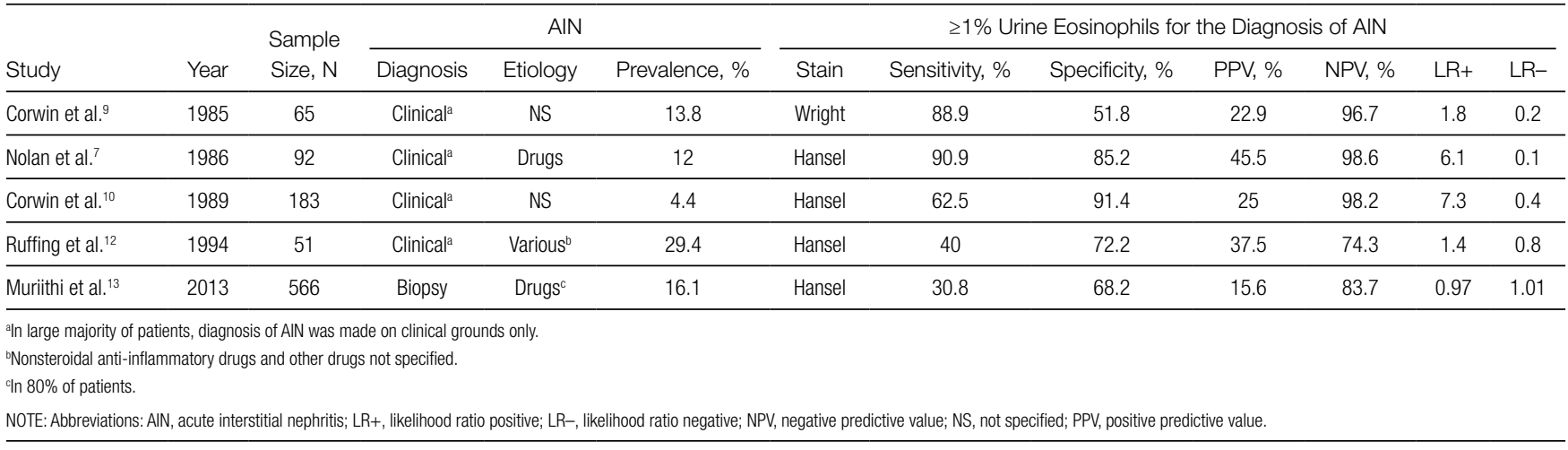

that the positive predictive value of UEs was inadequate in diagnosing AIN. Only 6 of their 15 patients with AIN had positive UEs. Urine eosinophils were also present in patients with other diagnoses (glomerulonephritis, chronic kidney disease, acute pyelonephritis, prerenal azotemia). Like many other investigators, Ruffing et al. made the AIN diagnosis on clinical grounds in the large majority of cases.

Muriithi et al. ${ }^{13}$ reported similarly negative results in their retrospective AKI study involving 566 Mayo Clinic patients and spanning almost 2 decades. The study included patients who underwent both Hansel-stain UE testing and kidney biopsy within a week of each other. Only $28(30 \%)$ of 91 biopsy-proven AIN cases were positive for UEs. Using the $1 \%$ cutoff for a positive UE test yielded only $30.8 \%$ sensitivity and $68.2 \%$ specificity. Using the $5 \%$ cutoff increased specificity to $91.2 \%$, at the expense of sensitivity $(19.2 \%)$; positive predictive value improved to only $30 \%$, and negative predictive value remained relatively unchanged, at $85.6 \%$. In short, Muriithi et al. found that UE testing had no utility in AIN diagnosis.

In summary, initial studies, such as those by Corwin et al, ${ }^{9,10}$ supported the conclusion that UEs are useful in AIN diagnosis but had questionable validity owing to methodologic issues, including small sample size and lack of biopsy confirmation of AIN. On the other hand, more recent studies, such as the one conducted by Muriithi et al., ${ }^{13}$ had larger sample sizes and biopsy-proven diagnoses and confirmed the poor diagnostic value of UEs in AIN.

The poor sensitivity and specificity of UE tests can have important consequences. A false positive test may cause the clinician to incorrectly diagnose the patient with AIN and prompt the clinician to remove medications that may be vitally important. The clinician may also consider treating the patient with steroids empirically. A false negative test may inappropriately reassure the clinician that the patient does not have AIN and does not need cessation of the culprit drug. This may also lead the clinician to forego a necessary kidney biopsy.

\section{WHAT YOU SHOULD DO INSTEAD}

A history of recent exposure to a classic offending drug (eg, beta-lactam, proton pump inhibitor, nonsteroidal anti-inflammatory drug) in combination with the classic triad of fever, rash, and peripheral eosinophilia suggests an AIN diagnosis. However, less than $5 \%$ to $10 \%$ of patients present with this triad. ${ }^{14,15}$ Regardless of the triad's presence, if other causes of AKI have been excluded, stopping a potential offending agent and monitoring for improvement are recommended. If a culprit drug cannot be safely discontinued, renal biopsy may be necessary for confirmation of the diagnosis. Moreover, if kidney function continues to deteriorate, a nephrology consultation may be warranted for guidance on the risks and benefits of performing a kidney biopsy to confirm the diagnosis and/ or the use of corticosteroids.

\section{RECOMMENDATIONS}

- Urine eosinophils should not be used in the diagnosis of AIN.

- The clinical diagnosis of drug-associated AIN should be based on excluding other possible likely etiologies of AKI and confirming the history of drug exposure. This is reinforced when kidney function improves upon discontinuation of offending agent.

- Kidney biopsy is the gold standard for AIN and should be performed if the clinical picture is unclear or the renal function is not improving upon discontinuation of offending agent.

\section{CONCLUSION}

Since the mid-1980s, studies have found that UEs are too insensitive and nonspecific to confirm or exclude the diagnosis of AIN in patients with AKI (Table). UEs are seen in other AKI etiologies, such as pyelonephritis, acute tubular necrosis, atheroembolic renal disease, and glomerulonephritis. Current evidence-based medicine does not support use of UEs as a biomarker for AIN. False-positive and false-negative results confuse the overall picture and result either in discontinuation of important medications and unnecessary steroid treatment or in delayed removal of a culprit medication. ${ }^{16}$

Our case's positive UE test does not affect the posttest probability that our patient has AIN. Presence of a culprit drug and absence of clinical data suggesting an alternative diag- 
nosis would lead most clinicians to change antibiotic therapy and observe for improvement in renal function.

Disclosure: Nothing to report.

Do you think this is a low-value practice? Is this truly a "Thing We Do for No Reason?" Share what you do in your practice and join in the conversation online by retweeting it on Twitter (\#TWDFNR) and Liking It on Facebook. We invite you to propose ideas for other "Things We Do for No Reason" topics by emailing TWDFNR@hospitalmedicine.org.

\section{References}

1. Wang HE, Muntner P, Chertow GM, Warnock DG. Acute kidney injury and mortality in hospitalized patients. Am J Nephrol. 2012;35(4):349-355.

2. Farrington K, Levison DA, Greenwood RN, Cattell WR, Baker LR. Renal biopsy in patients with unexplained renal impairment and normal kidney size. Q J Med. 1989;70(263):221-233.

3. Michel DM, Kelly CJ. Acute interstitial nephritis. J Am Soc Nephrol. 1998;9(3):506-515.

4. Neilson EG. Pathogenesis and therapy of interstitial nephritis. Kidney Int. 1989;35(5):1257-1270.

5. Galpin JE, Shinaberger JH, Stanley TM, et al. Acute interstitial nephritis due to methicillin. Am J Med. 1978;65(5):756-765.
6. Linton AL, Clark WF, Driedger AA, Turnbull DI, Lindsay RM. Acute interstitial nephritis due to drugs: review of the literature with a report of nine cases. Ann Intern Med. 1980;93(5):735-741

7. Nolan CR 3rd, Anger MS, Kelleher SP. Eosinophiluria-a new method of detection and definition of the clinical spectrum. N Engl J Med. 1986;315(24): 1516-1519.

8. Perazella MA, Bomback AS. Urinary eosinophils in AIN: farewell to an old biomarker? Clin J Am Soc Nephrol. 2013;8(11):1841-1843.

9. Corwin HL, Korbet SM, Schwartz MM. Clinical correlates of eosinophiluria. Arch Intern Med. 1985;145(6):1097-1099.

10. Corwin HL, Bray RA, Haber MH. The detection and interpretation of urinary eosinophils. Arch Pathol Lab Med. 1989;113(11):1256-1258.

11. Sutton JM. Urinary eosinophils. Arch Intern Med. 1986;146(11):2243-2244.

12. Ruffing KA, Hoppes P, Blend D, Cugino A, Jarjoura D, Whittier FC. Eosinophils in urine revisited. Clin Nephrol. 1994;41(3):163-166.

13. Muriithi AK, Nasr SH, Leung N. Utility of urine eosinophils in the diagnosis of acute interstitial nephritis. Clin J Am Soc Nephrol. 2013;8(11):1857-1862.

14. Clarkson MR, Giblin L, O'Connell FP, et al. Acute interstitial nephritis: clinical features and response to corticosteroid therapy. Nephrol Dial Transplant. 2004;19(11):2778-2783.

15. Rossert J. Drug-induced acute interstitial nephritis. Kidney Int. 2001;60(2): 804-817.

16. Fletcher A. Eosinophiluria and acute interstitial nephritis. $N$ Engl J Med. 2008;358(16):1760-1761. 